\title{
Molecular analysis of faecal and duodenal samples reveals significantly higher prevalence and numbers of Pseudomonas aeruginosa in irritable bowel syndrome
}

Correspondence

Angèle P. M. Kerckhoffs

AngeleKerckhoffs@hotmail.com

Received 9 June 2010

Accepted 8 October 2010

\author{
Angèle P. M. Kerckhoffs, ${ }^{1}$ Kaouther Ben-Amor, ${ }^{2}$ Melvin Samsom, ${ }^{1}$ \\ Michel E. van der Rest, ${ }^{3}$ Joris de Vogel, ${ }^{3}$ Jan $\mathrm{Knol}^{2}$ \\ and Louis M. A. Akkermans ${ }^{1}$ \\ ${ }^{1}$ Gastrointestinal Research Unit, Departments of Gastroenterology and Surgery, University Medical \\ Center Utrecht, Utrecht, The Netherlands \\ ${ }^{2}$ Danone Research - Centre for Specialised Nutrition, Wageningen, The Netherlands \\ ${ }^{3}$ BioVisible BV, Groningen, The Netherlands
}

\section{INTRODUCTION}

Irritable bowel syndrome (IBS) is a gastrointestinal disorder of unknown aetiology characterized by abdominal pain and change in bowel habit. Alteration in faecal microbiota composition and abnormal colonic fermentation imply that gastrointestinal microbiota may play a role in the pathogenesis of IBS (Spiller, 2007; Quigley, 2007). In $7-30 \%$ of IBS patients, acute gastrointestinal infection has been proposed as trigger of the onset of IBS symptoms, and the inflammatory response to the infection may cause persistent sensory-motor dysfunction (Madden \& Hunter, 2002; Rodríguez \& Ruigómez, 1999; Parry et al., 2003; Collins et al., 2001). Moreover, antibiotic therapy and

Abbreviations: DGGE, denaturing gradient gel electrophoresis; IBS, irritable bowel syndrome; q-PCR, quantitative real-time PCR. probiotic supplementation have been shown to reduce IBS symptoms in a subset of IBS patients, by either eradication of small intestinal bacterial overgrowth or modulation of the composition of the microbiota (Pimentel et al., 2000; Nobaek et al., 2000; Niedzielin et al., 2001; O'Mahony et al., 2005). Antibiotic therapy may also be a risk factor for developing IBS symptoms due to changes in bowel microbiota and colonization of pathogenic bacteria (Maxwell et al., 2002). It is well recognized that the microbial community is significantly altered in IBS (King et al., 1998; Posserud et al., 2007). Conventional culturing methods have shown that faeces of IBS patients contain higher numbers of facultatively anaerobic bacteria and members of the Enterobacteriaceae and lower numbers of lactobacilli and bifidobacteria than those of healthy subjects (Malinen et al., 2005; Balsari et al., 1982; Madden \& Hunter, 2002; Si et al., 2004). 
Detection and identification of gut bacteria by culturing is hampered by the limitations of culture conditions, and a large number of intestinal bacteria cannot be cultured (Mai \& Morris, 2004; Furrie, 2006; Suau et al., 1999). Molecular techniques based on 16S rRNA gene analysis allow a more comprehensive assessment of this complex microbial ecosystem (Vaughan et al., 2000). Using PCR-based techniques, a significant difference in the predominant faecal groups has been reported in IBS patients (Malinen et al., 2005; Kassinen et al., 2007). Studies investigating the gut microbiota in IBS using molecular-based methods have been limited to the faecal microbiota and have not assessed mucosa-associated microbiota in the small intestine. The mucosa-associated bacteria are thought to have a stronger interaction than luminal bacteria with the host (Hooper \& Gordon, 2001). The composition of luminal and mucosaassociated bacteria is not the same since the microenvironments are very different at the surface of the intestinal epithelium than in the lumen (Zoetendal et al., 2002; Lepage et al., 2005).

The aim of our research was to determine differences in mucosa-associated small intestinal and luminal faecal microbiota between IBS patients and healthy subjects using a molecular approach based on the sequence variability of the $16 \mathrm{~S}$ rRNA gene.

\section{METHODS}

Subjects. Thirty-seven IBS patients who fulfilled Rome II criteria for IBS were included in this study. The IBS patient group consisted of 13 alternating IBS patients, 13 diarrhoea-predominant IBS patients and 11 constipation-predominant IBS patients. Twenty healthy subjects (control group) had neither intestinal complaints nor a history of bowel resection. All subjects had to stop antibiotics at least 1 month before endoscopy. Use of probiotics, proton pump inhibitors and antisecretory medication had to be stopped at least 3 weeks prior to endoscopy. The standard preparation for an upper endoscopy in our hospital is fasting $12 \mathrm{~h}$ before the endoscopy. At the request of a subject, Xylocaine spray is used to anaesthetize the throat or a sedative (Dormicum $5 \mathrm{mg}$ ) is given. The Human Ethics Committee of the University Medical Centre Utrecht approved the study and all subjects gave written informed consent.

Sampling, preparation and storage. A sterile cytology brush (Uno-Brush; Prince Médical) sheathed in a sterile catheter was placed through the endoscope biopsy channel and advanced under direct vision out beyond the endoscope tip (León-Barúa et al., 1993). The duodenal mucosa was brushed three times to obtain mucosaassociated bacteria. Brush samples from each subject were obtained from the descending and horizontal part of the duodenum. After brushing, the brush was pulled back into the sheath of the catheter, which was removed and the brush was immediately cut off the catheter and placed into a sterile tube in liquid nitrogen and stored at $-80{ }^{\circ} \mathrm{C}$ until analysis.

Faecal samples were collected before the endoscopy and were stored at $-80{ }^{\circ} \mathrm{C}$ until analysis. Both faecal and brush samples were shipped in dry ice to the microbiological laboratory for analysis.

DNA extraction and PCR amplification. Faecal samples were thawed and DNA extraction was performed using the Fast DNA Spin kit (Qbiogene) from approximately $0.1 \mathrm{~g}$ faecal material. The frozen brush samples were thawed and suspended in $180 \mu$ ATL buffer (Qiagen) and vigorously vortexed to extract the attached bacteria. Subsequently, DNA was isolated from the mucosa-associated bacteria using the DNeasy Blood \& Tissue kit (Qiagen) according to the manufacturer's instructions. The isolated DNA solutions from both faeces and brush samples were stored at $-20{ }^{\circ} \mathrm{C}$.

The extracted DNA was used as a template to amplify the V6-V8 regions of the 16S rRNA gene with primers U968-GC-f (5' -CGC CCG GGG CGC GCC CCG GGC GGG GCG GGG GCA CGG GGG GAA CGC GAA GAA CCT TAC-3'), which contains at its $5^{\prime}$-end a 40-base GC clamp, and U1401-r (Muyzer et al., 1993; Muyzer \& Smalla, 1998). PCRs were performed using a Taq DNA polymerase kit from Invitrogen. The reaction mixture consisted of $1 \times$ PCR buffer, $3 \mathrm{mM}$ $\mathrm{MgCl}_{2}, 50 \mu \mathrm{M}$ each deoxynucleotide triphosphate, $1.25 \mathrm{U}$ Taq polymerase, $10 \mathrm{pmol}$ each primer and $1 \mu \mathrm{l}$ appropriately diluted template DNA in a final volume of $50 \mu \mathrm{l}$. Samples were amplified in a PTC-200 PCR system (MJ Research) with the following thermocycling program: $94{ }^{\circ} \mathrm{C}$ for $5 \mathrm{~min} ; 10$ cycles of denaturation at $94{ }^{\circ} \mathrm{C}$ for $1 \mathrm{~min}$, annealing at $65-56{ }^{\circ} \mathrm{C}$ for $1 \mathrm{~min}$ (reduction of $1{ }^{\circ} \mathrm{C}$ for each cycle) and extension at $68^{\circ} \mathrm{C}$ for $3 \mathrm{~min} ; 33$ cycles for faecal and 43 cycles for brush samples of $94{ }^{\circ} \mathrm{C}$ for $1 \mathrm{~min}, 56{ }^{\circ} \mathrm{C}$ for $1 \mathrm{~min}$ and $68{ }^{\circ} \mathrm{C}$ for $3 \mathrm{~min}$. Aliquots of $5 \mu \mathrm{l}$ PCR product were analysed by electrophoresis on a $1.5 \%(\mathrm{w} / \mathrm{v})$ agarose gel containing ethidium bromide $(0.044 \%$, v/v).

Denaturing gradient gel electrophoresis analysis of PCR amplicons. PCR amplicons were separated by denaturing gradient gel electrophoresis (DGGE) based on the protocol of Muyzer \& Smalla (1998) using the DGGE Decode system (Bio-Rad Laboratories) with the following modifications. Polyacrylamide gels consisted of $8 \%(\mathrm{v} / \mathrm{v})$ polyacrylamide (ratio of acrylamide/bisacrylamide, $37.5: 1$ ) and $0.5 \times$ Tris/acetate-EDTA (TAE; $\mathrm{pH}$ 8.0) buffer. Denaturing acrylamide of $100 \%$ was defined as $7 \mathrm{M}$ urea and $40 \%$ formamide. The polyacrylamide gels were made with a denaturing gradient ranging from 32.5 to $72.5 \%$. The gels were poured from the top using a gradient maker and a pump (Econo gradient pump; BioRad Laboratories) set at a speed of $3 \mathrm{ml} \mathrm{min}^{-1}$. Prior to the polymerization of the denaturing gel (gradient volume, $28 \mathrm{ml}$ ), a $7.5 \mathrm{ml}$ stacking gel without denaturing chemicals was added, and the appropriate comb was subsequently inserted. Electrophoresis was performed first for $5 \mathrm{~min}$ at $200 \mathrm{~V}$ and then at $80 \mathrm{~V}$ for $17 \mathrm{~h}$ in $0.5 \times$ TAE buffer at a constant temperature of $60{ }^{\circ} \mathrm{C}$. The gels were stained with $\mathrm{AgNO}_{3}$ as described by Sanguinetti et al. (1994) and dried overnight at $50{ }^{\circ} \mathrm{C}$.

Analysis of the DGGE profiles and generation of pooled profiles. The average curve feature 'show average curve' of GelCompar software (Applied Maths) was used to create averaged fingerprints. This script generates an averaged fingerprint using the densitometric and location results from the bands in the original gels. The intensity of the band in the average IBS patient or healthy subject profile is calculated by the intensity of the original bands in the original gels of all IBS patients or healthy subjects, respectively. From all of the DGGE gels from the IBS patient samples we created the averaged IBS profile and using all of the DGGE gels from the healthy subject samples we created the healthy subjects averaged profile. Percentage similarity between the averaged IBS profile and the averaged healthy subject profile is based on the number of identical bands and the numeric values of the band intensity (with $5 \%$ background subtraction) found in the two profiles. Similar band positions are removed, generating a graph with unique bands for healthy subjects and unique bands for IBS patients. Band differences in these average computer-generated profiles were determined and the bands which were unique for either group were localized in the individual original DGGE profiles. The most intense bands in the individual profiles were cut from the original gels. The DNA from 
(a)

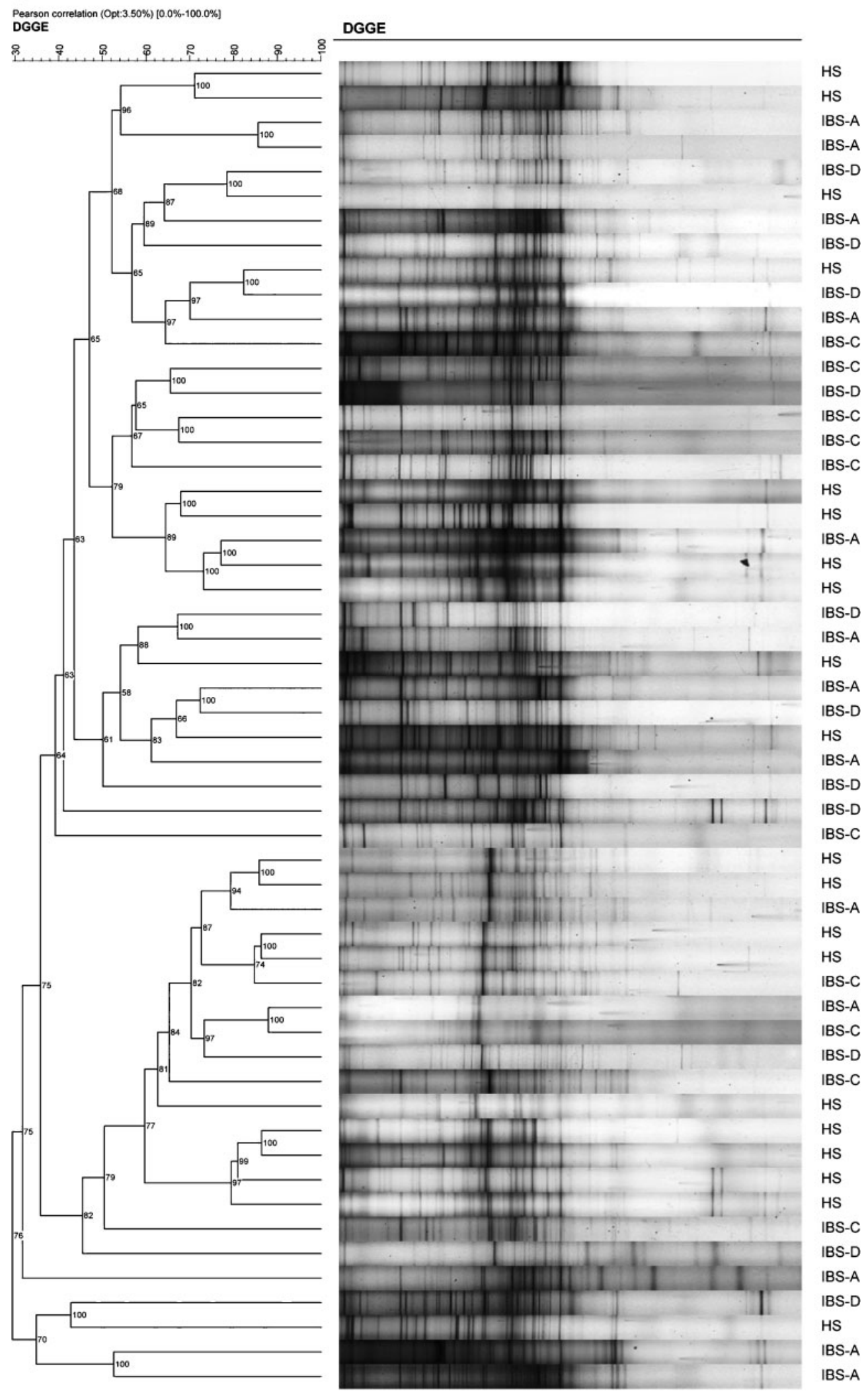

Fig. 1. DGGE analysis of the duodenal mucosa-associated and faecal bacteria. No evident clusters were shown in the clustering tree of the silver-stained DGGE gel of the V6-V8 regions of the 16S rRNA gene, obtained from duodenal samples (a) and faecal samples (b) of 20 healthy subjects (HS), 11 diarrhoea-predominant IBS patients (IBS-D), 13 alternating IBS patients 
(b)

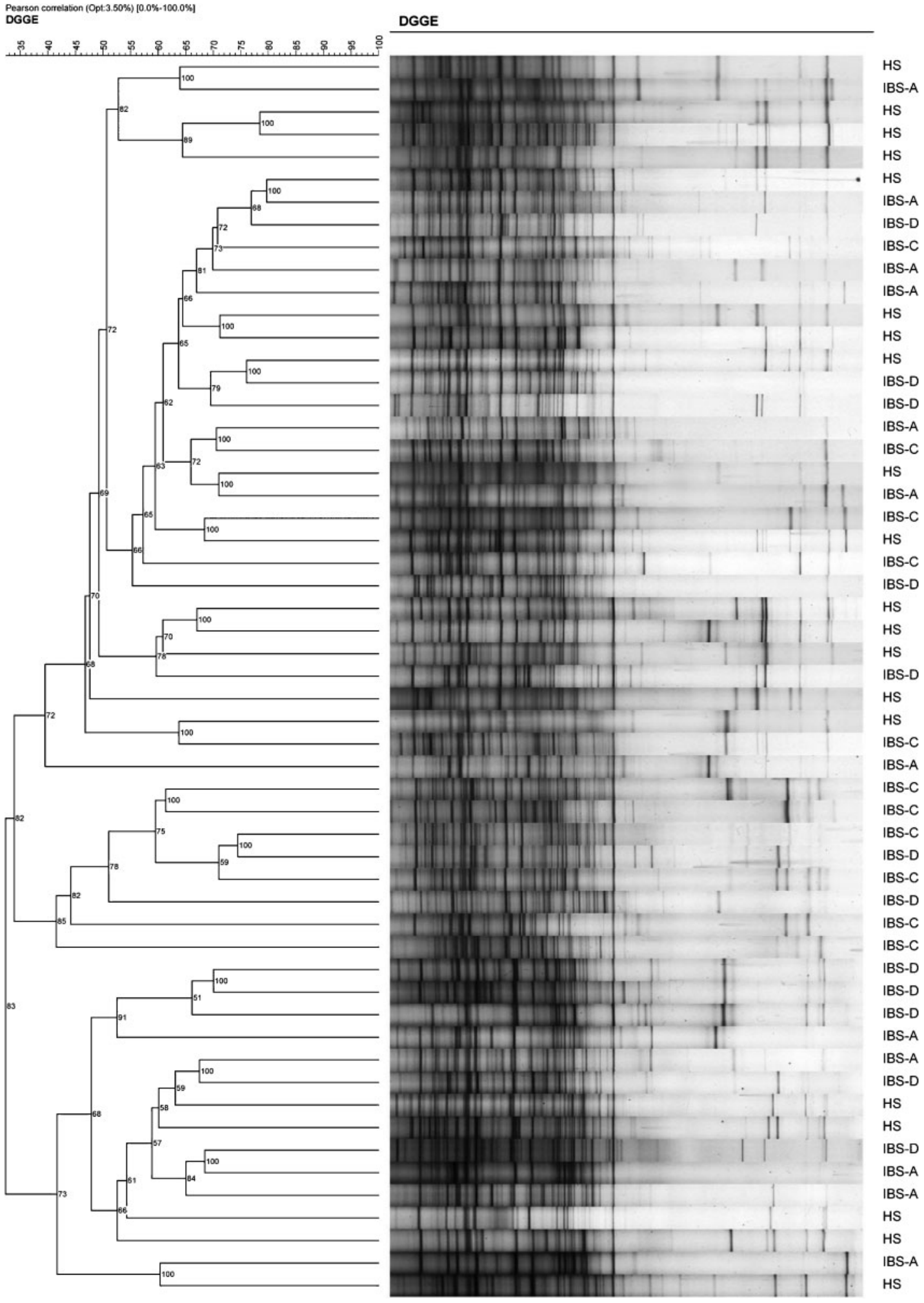

(IBS-A) and 10 constipation-predominant IBS patients (IBS-C). Dendrograms based on the DGGE gel for each individual were generated by using the Pearson correlation index for each pair of lanes within a gel and were used as a measure of similarity between the fingerprints. The scale bar indicates the DGGE similarity between profiles. The clustering of patterns was calculated using the unweighted-pair group method using average linkages. 
these bands was used for a second DGGE analysis using a 46-51.6\% gradient. Bands of interest were subjected to sequencing as described below.

DGGE band sequence analysis. To identify the bacteria, a small piece from the middle of the selected band was cut from the DGGE gel with a sterile scalpel and then incubated in $50 \mu$ sterile MilliQ water for $24 \mathrm{~h}$ at $4{ }^{\circ} \mathrm{C}$ to allow diffusion. The eluent containing DNA fragments was used for PCR reamplification with the same primers used earlier. To check whether the DNA of interest on the first DGGE gel and the reamplified DNA migrated to the same position, we carried out a second DGGE in order to compare the two samples. When the two bands co-migrated, DNA fragments were purified with the GenElute PCR DNA Purification kit (Sigma) and thereafter ligated into the pCR2.1-TOPO vector and transformed into Escherichia coli One Shot TOP10 competent cells (Invitrogen). Plasmids from colonies of kanamycin-resistant transformants were extracted with the Qiagen Plasmid Midi Purification kit. The extracted plasmids were screened for inserts of the correct size by performing a PCR with the M13 forward and reverse primers as well as with the $968 \mathrm{f}$ and $1401 \mathrm{r}$ primers. Insert PCR amplicons of selected transformants were purified and subjected to DNA sequence analysis (Baseclear). Sequence similarities of the clones were checked with the Basic Local Alignment Search Tool (BLAST) at the NCBI database (http://www.ncbi.nlm.nih.gov/BLAST) (Altschul et al., 1990).

Quantitative real-time PCR analysis. Quantitative real-time PCR (q-PCR) was performed to determine the percentage of Pseudomonas aeruginosa in brush and faecal samples. Measurement of $P$. aeruginosa was performed as described by Pirnay et al. (2000) with some slight modifications. The detection limit of the assay was $10^{3}$ c.f.u. $\mathrm{g}^{-1}$ and 1-10 c.f.u. per reaction (Pirnay et al., 2000). The fluorescent labels were changed from LC Red 640 to 6FAM and from fluorescein to TAMRA while the other ingredients and conditions for the described q-PCR stayed the same. The fluorescence signal was measured in the annealing phase on the ABI 7900HT Fast system (Applied Biosystems). The total bacterial load was determined as described by Nadkarni et al. (2002). The relative percentage of $P$. aeruginosa was subsequently calculated according to Liu \& Saint (2002a, b). The efficiency of each amplification curve was calculated separately and used to determine the initial relative amounts of DNA. The amount used was in general between 100 and 200 ng DNA per reaction. Finally, the obtained ratios between the initial amounts of DNA were normalized against a monoculture of the same species, which was set at $100 \%$.

Statistical analysis. DGGE data were analysed by GelCompar II software (Applied Maths). Cluster analysis and calculation of the similarity indices between the different banding patterns were performed using Pearson product-moment correlation and the unweighted-pair group method using arithmetic averages.

$P$. aeruginosa levels in IBS patients and healthy subjects were analysed using independent samples $t$-test. $P$. aeruginosa levels in IBS subgroups were analysed using one-way ANOVA with Bonferroni correction. Data are expressed as mean \pm SEM. SPSS 12.0.1 for Windows was used for analysis.

\section{RESULTS}

\section{Study population}

Healthy subjects (5 men, 15 women) were significantly ( $P=0.005)$ younger $(32 \pm 2.6$ years $)$ than the IBS patients (11 men, 26 women; $42 \pm 2.3$ years). No differences between both groups in gender distribution were observed.

\section{Bacterial diversity of the dominant microbiota as assessed by DGGE}

DGGE analysis of the PCR-amplified fragments of the V6V8 region of the 16S rRNA gene of both duodenal mucosaassociated bacteria (Fig. 1a) and faecal bacteria (Fig. 1b) from IBS patients and healthy subjects was performed, showing no evident clusters between healthy subjects and IBS subgroup patients. Comparing the fingerprints of faecal and small intestinal samples for all subjects, a clear clustering was observed independently from the disease state (data not shown), indicating that the mucosaassociated microbiota is significantly different from the faecal microbiota, as also observed in a previous study (Zoetendal et al., 2002). Based on the DGGE profiles, no clear difference between the IBS patients and healthy subjects could be detected for the most abundant microbiota at any sampling location. Moreover, statistical analysis of the DGGE profiles from both sampling sites (duodenal brush and faecal samples) did not reveal any specific core microbiota that could be distinguished between healthy subjects, IBS patients or IBS subgroup patients. However, some subclusters could be identified, suggesting overlap of specific microbial components. To identify possible microbial components specifically associated with healthy subjects or with IBS patients, the DGGE profiles generated from all IBS patients and those from healthy subjects were pooled in average profiles (Figs 2 and 3).

Comparing the generated average duodenal fingerprints of healthy subjects and IBS patients, $14(78.2 \%)$ of the bands were identical for both groups, 6 bands were specific for IBS patients ( $10 \%$ of all IBS bands) while 27 bands were specific for healthy subjects (34\% of all healthy subject bands) (see Fig. 2). The band location and intensity in the average profiles were used to generate Fig. 2.

Fig. 3 shows the average faecal fingerprints of both groups, in which $21(86.25 \%)$ of the bands were identical, 33 bands were confined to healthy subjects $(21 \%$ of all healthy subject bands) and 17 bands were confined to IBS patients (10\% of all IBS bands). The DGGE band fragments that were of interest were further characterized by sequence analysis.

\section{Sequence analysis of the dominant microbiota}

The 16S rRNA genes from the isolated bands from the DGGE profiles of faecal and duodenal samples from the healthy subjects and IBS patients were amplified and cloned in E. coli. The V6-V8 region of the cloned $16 \mathrm{~S}$ rRNA gene fragments from the cell lysates of transformants was amplified. In this way, 51 clones were generated, the plasmid DNA from the corresponding clones was purified and, subsequently, the nucleotide sequence inserts were determined and compared to the 16S rRNA databases using the BLAST tool (Zhang et al., 2000). From the duodenal samples, a total of 19 clones, comprising 15 clones recovered from 4 unique bands from the IBS 
patients and 4 clones from 1 unique band from the healthy subjects, were generated. A total of 32 clones retrieved from faecal samples, comprising 24 clones from 3 unique bands from IBS patients and 8 clones from 1 unique band from healthy subjects, were obtained.

Among the 51 sequenced clones retrieved from both groups, 35 shared $95 \%$ or less $16 \mathrm{~S}$ rRNA sequence identity with their nearest relatives based on a BLAST search. This indicated that the majority of the sequences were derived from new, as yet undescribed, phylotypes. Most cloned sequences retrieved from the IBS patients could be assigned to the class Gammaproteobacteria mainly to Pseudomonas species, and other clones retrieved essentially from the faecal samples were assigned to Clostridium nexile. However, in the healthy subjects, the cloned sequences were allocated to the two major phylotypes commonly encountered in human faecal clone libraries, namely Clostridium cluster XIVa and Bacteroidetes.

Brush samples. Out of the 15 clones retrieved from the brush samples from IBS patients, seven cloned sequences were affiliated with different Pseudomonas species with a sequence similarity ranging from 94 to $99 \%$. Remarkably, one cloned sequence was almost identical to $P$. aeruginosa ( $99 \%$ sequence similarity). Three clones were closely related to Klebsiella pneumoniae with $96-97 \%$ sequence similarity, one clone was almost identical to Oribacterium sinus (99\% sequence similarity) and there were two new phylotypes (90-96\% sequence similarity to an uncultured bacterium clone, rc2-18, isolated from rat faeces; Brooks et al., 2003). In addition, one clone showed $96 \%$ sequence similarity with an uncultured Neisseria sp. clone isolated from $P$. aeruginosa-colonized patients (Flanagan et al., 2007) and shared only $91 \%$ similarity with the closest cultured relative, i.e. Kingella kingae strain ATCC 23330. Another clone was related to uncultured Neisseria sp. clone $101 \mathrm{C} 07$ isolated from the oral cavity with only $91 \%$ sequence similarity.

The four cloned sequences obtained from the healthy subjects were identified as Serratia sp., Acinetobacter sp., Pantoea sp. and an uncultured Clostridiales bacterium with 99, 98, 91 and $95 \%$ sequence similarity, respectively.

Faecal samples. Out of the 24 clones retrieved from faecal samples from IBS patients, 11 cloned sequences were affiliated with different Pseudomonas species with a sequence similarity ranging from 85 to $100 \%$. Noticeably, one cloned sequence showed a perfect match with $P$. aeruginosa and three other sequences shared 88-95\% similarity with the same species. Furthermore, eight clones were affiliated with different Clostridium species. Among these, six clones were closely related to $C$. nexile with sequence similarity varying from 93 to $97 \%$, and two clones were identified as Clostridium sp. with sequence similarity varying from $92 \%$ to $96 \%$. One clone was identified as Desulfovibrio vulgaris ( $98 \%$ similarity), and one clone was related to an uncultured faecal bacterium clone isolated by
Li et al. (2008) with $94 \%$ sequence similarity. In addition, two clones shared only $92 \%$ and $91 \%$ sequence similarity with Burkholderia mallei and finally one cloned sequence was related to Alcaligenes faecalis with $92 \%$ sequence similarity.

Out of the eight clones from the faecal samples from healthy subjects, five cloned sequences were affiliated with different Bacteroides species with a sequence similarity ranging from 95 to $99 \%$, with the closest culturable species being Bacteroides ovatus ( $97 \%$ sequence similarity), Bacteroides vulgatus ( $96 \%$ sequence similarity) and Bacteroides coprophilus ( $99 \%$ sequence similarity). Furthermore, one clone was almost identical to Prevotella corporis ( $99 \%$ sequence similarity), one clone shared $95 \%$ sequence similarity with Prevotella oulorum and one clone shared $97 \%$ sequence similarity with Clostridium butyricum.

\section{Quantification of $\boldsymbol{P}$. aeruginosa using real-time PCR}

Since the majority of IBS unique bands belonged to the genus Pseudomonas, of which $P$. aeruginosa was the most frequently identified species, real-time PCR was used to quantify $P$. aeruginosa in the brush and faecal samples.

The data in Fig. 4(a) show that the relative abundance of $P$. aeruginosa (\% of total bacterial load) was significantly $(P<0.001)$ higher in IBS patients $(8.3 \% \pm 0.950)$ than in healthy subjects $(0.1 \% \pm 0.069)$. The $C_{\mathrm{t}}$ value of the positive controls, negative control (water), all IBS patients and healthy controls was $<22,>45,30.4$ (SEM 0.84 ) and 36.6 (SEM 0.83), respectively. Moreover, the prevalence of $P$. aeruginosa in the duodenal samples was $97.3 \%$ in IBS patients and only $40 \%$ in healthy subjects.

The results illustrated in Fig. 4(b) reveal that the percentage of $P$. aeruginosa in faecal samples was also significantly higher $(P<0.001)$ in IBS patients $(2.34 \% \pm 0.31)$ than in healthy subjects $(0.003 \% \pm 0.0027)$. We also found that $P$. aeruginosa was detected in $97.2 \%$ of the faecal samples from IBS patients whereas only $15.8 \%$ of the healthy subjects were positive carriers of $P$. aeruginosa. No differences related to the abundance and prevalence of $P$. aeruginosa between IBS subgroups were detected in both sampling sites.

\section{DISCUSSION}

This study shows clearly that, analysing the entire faecal and small intestinal microbiota population using DGGE, there are specific DGGE bands for healthy subjects and specific bands for IBS patients. Focussing on the specific bands showed that most clones belonged to the genus Pseudomonas, of which $P$. aeruginosa was the predominant species. The most important finding of this study is the higher prevalence and levels of $P$. aeruginosa 


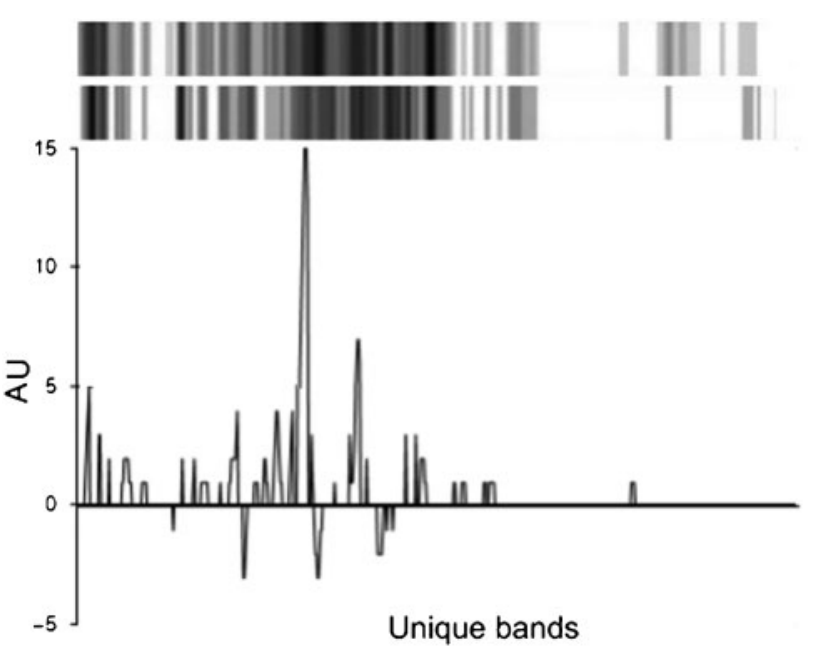

HS

IBS in small intestinal and faecal samples from IBS patients than in those from healthy subjects, analysed by q-PCR.

Previously, Kassinen et al. (2007) also showed distinct microbiota based on their mol\% G $+\mathrm{C}$ fractions in faecal samples from IBS subtypes and from controls using molecular tools. After faecal microbial genomes of IBS subtypes and controls were pooled, differences in the Firmicutes, Bacteroidetes, Actinobacteria and Proteobacteria were shown (Kassinen et al., 2007). Our sequence analysis data showed that members of the Proteobacteria and Firmicutes were specific for IBS patients. Pseudomonas sp. was the most prevalent and q-PCR analysis showed a higher prevalence and levels of $P$. aeruginosa in small intestinal mucosa-associated and faecal samples from IBS patients than in those from healthy subjects.

P. aeruginosa, a Gram-negative aerobic rod, is known to predominantly infect immunosuppressed and cystic fibrosis patients (Oliver et al., 2000; Fichtenbaum et al., 1994; Baron \& Hollander, 1993). The $15.8 \%$ prevalence of
Fig. 2. Average DGGE profiles of all fingerprints from IBS patients $(n=34)$ and all fingerprints from healthy subjects $(n=20)$ in duodenal samples constructed using GelCompar II software. IBS represents the average of 34 IBS patients and $\mathrm{HS}$ represents the average profile of 20 healthy subjects. The graph shows a numerical presentation of the average profile with the unique bands present in the healthy subjects on top and the unique bands for the IBS group on the bottom of the graph. AU, Arbitrary units.
P. aeruginosa in faecal samples from healthy subjects in this study is within the range of $0-24 \%$ reported in other studies (Bonten et al., 1999; Speert et al., 1993; Kessner \& Lepper, 1967; Bleday et al., 1993; Levy, 2000). Higher prevalence $(97 \%)$ and levels of $P$. aeruginosa in IBS patients have not been reported in the literature to our knowledge. However, in functional dyspepsia patients, Pseudomonas was cultured as the predominant bacterium in the small intestine (León-Barúa et al., 1993). Previous studies using molecular-based methods to determine the quantity of faecal microbiota in IBS patients and healthy subjects showed differences in microbiota composition (Kassinen et al., 2007; Malinen et al., 2005). However, $P$. aeruginosa levels were not tested in these studies.

The age differences between the healthy subjects and IBS patients in our study might be a confounding factor. The effect of the age difference between healthy subjects (mean age 32 years) and IBS patients (mean age 42 years) on bacterial DNA load, prevalence and quantity of $P$. aeruginosa is not known. Current literature does describe change in faecal microbiota composition in subjects older

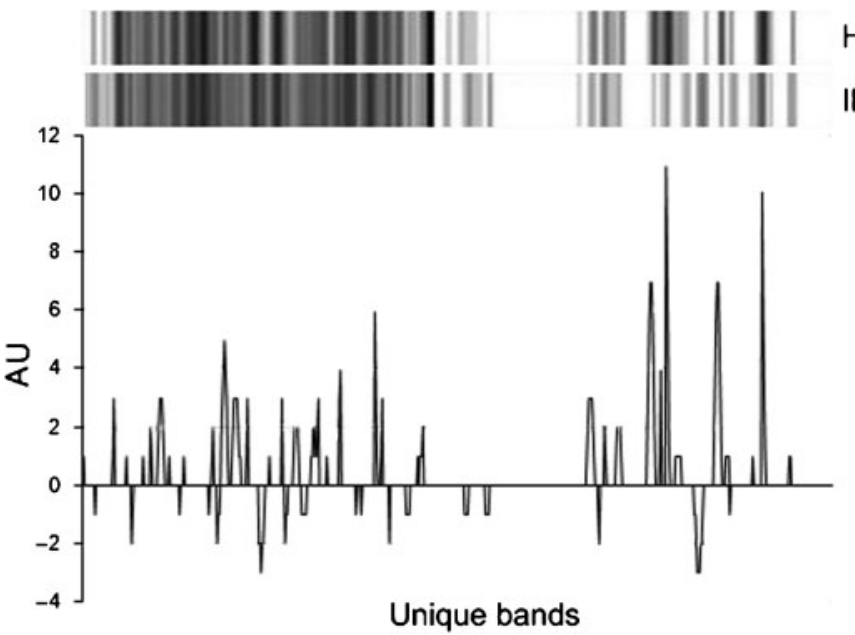

HS

IBS
Fig. 3. Average DGGE profiles of all fingerprints from IBS patients $(n=34)$ and all fingerprints from healthy subjects $(n=20)$ in faecal samples constructed using GelCompar II software. IBS represents the average of 34 IBS patients and HS represents the average profile of 20 healthy subjects. The graph shows a numerical presentation of the average profile with the unique bands present in the healthy subjects on top and the unique bands for the IBS group on the bottom of the graph. $\mathrm{AU}$, Arbitrary units. 


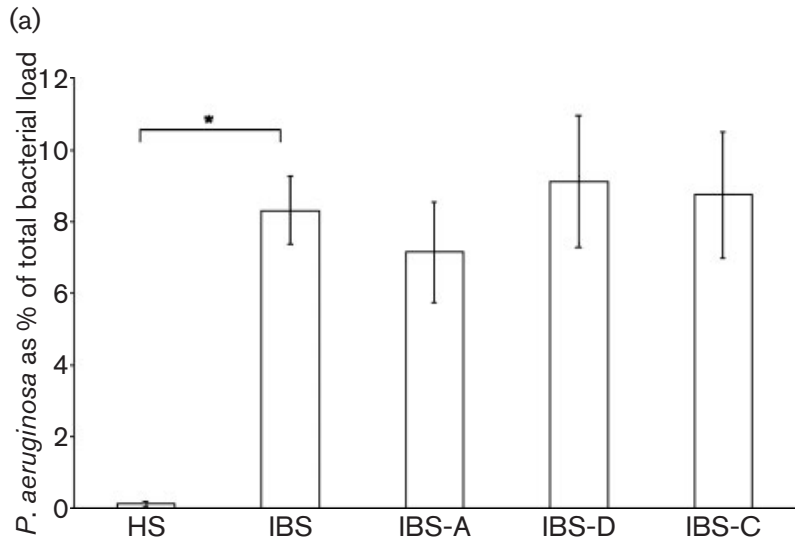

(b)

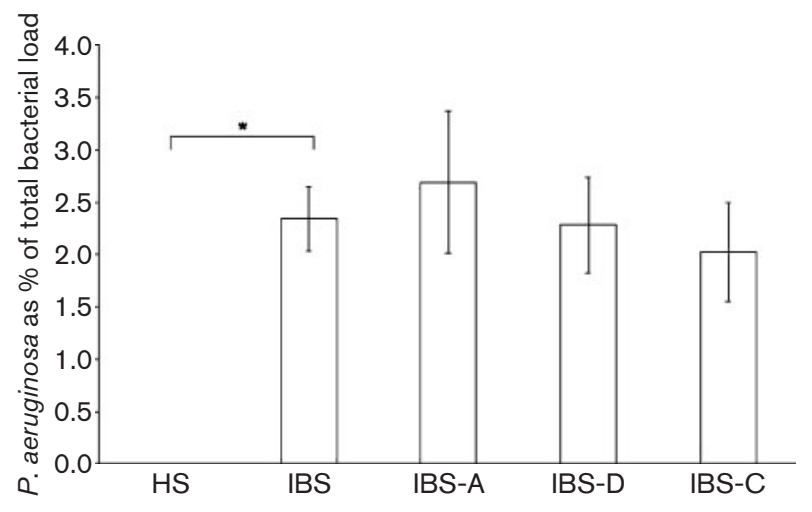

Fig. 4. Duodenal $P$. aeruginosa as a percentage of total bacterial load of healthy subjects (HS), IBS patients (IBS) and the IBS subgroups [alternating IBS patients (IBS-A), diarrhoea-predominant IBS patients (IBS-D) and constipation-predominant IBS patients (IBS-C)] in duodenal samples (a) or faecal samples (b). Values are represented as means \pm SEM. ${ }^{*} P<0.001$.

than 65 years of age compared to younger adults (Hopkins \& Macfarlane, 2002; Woodmansey et al., 2004; Mueller et al., 2006). To evaluate the effect of age difference in adult subjects on microbiota composition, further investigations are needed.

The question arises whether differences in prevalence and quantity of $P$. aeruginosa might be epiphenomenal or be the cause of IBS symptoms. First of all, it is known that $P$. aeruginosa may occur as the sole potential pathogen in patients with diarrhoea and $P$. aeruginosa induces signs and symptoms of enteritis in antibiotic-treated rats (Adlard et al., 1998). Also, a causative relationship between $P$. aeruginosa and diarrhoea has been found in immunocompromised individuals (Adlard et al., 1998). However, in our study, $P$. aeruginosa was increased not only in diarrhoea-predominant IBS patients, but also in constipation-predominant and alternating IBS patients.

Secondly, proteases of $P$. aeruginosa are known to disable protease-activated receptor-2 (PAR-2) in the respiratory tract (Dulon et al., 2005). The effect of $P$. aeruginosa proteases on PAR-2 in the gastrointestinal tract is unknown. PAR-2 activation in the gastrointestinal tract has been shown to modify motility patterns, inflammatory mediator release, intestinal barrier integrity, ion transport and nociceptive functions, all functions which are part of gut physiology involved in generation of IBS symptoms (Vergnolle, 2005).

Indication that increased $P$. aeruginosa might be epiphenomenal is reported in a recent study which showed high levels of Pseudomonas species in pouchitis patients in remission. If the normal microbiota of the gastrointestinal tract is altered, potential pathogens can colonize or resident pathogens can multiply. The remission of the pouchitis patients was induced by antibiotic therapy (Kühbacher et al., 2006). It has been shown that antibiotic treatment of mice increases the colonization potential of Pseudomonas species (George et al., 1989). P. aeruginosa is naturally resistant to many antibiotics due to the permeability barrier of its outer membrane lipopolysaccharide, tendency to colonize surfaces in a biofilm form and maintenance of antibiotic-resistance plasmids (Mah et al., 2003; Walters et al., 2003; Masuda et al., 1999; Livermore, 1992). Antibiotics and probiotics used more than a month before the start of the study are unlikely to have had an effect on the $P$. aeruginosa colonization since stabilization of the intestinal microbiota within 1-2 weeks after antibiotic therapy has been reported (Woodmansey et al., 2004; Edlund \& Nord, 2000; Hentges et al., 1985; Levison, 1973; Gorbach et al., 1988).

In a previous study, we showed a lower Bifidobacterium catenulatum level in both faecal and duodenal brush samples from IBS patients than in those from healthy subjects (Kerckhoffs et al., 2009). This decrease in normal microbiota might give $P$. aeruginosa the opportunity to multiply. A major obstacle in relating microbial composition with a role in IBS pathophysiology is the fact that each individual is inhabited by a specific microbial community shaped by host and environmental factors (Eckburg et al., 2005).

In conclusion, our data show no apparent difference in the diversity of predominant microbiota profiles between IBS patients and healthy subjects. However, using the pooled average profiles of the PCR-DGGE fingerprints allowed us to isolate and sequence specific bands from IBS patients and healthy subjects. IBS-specific bands were predominantly members of the genus Pseudomonas. q-PCR analysis confirmed that $P$. aeruginosa is found more frequently and in higher levels in IBS patients than in healthy subjects. Therefore, we propose that $P$. aeruginosa may be involved in the pathophysiology of IBS.

\section{ACKNOWLEDGEMENTS}

The authors thank Monique Haarman and Eric Caldenhoven for their contribution to the study. 


\section{REFERENCES}

Adlard, P. A., Kirov, S. M., Sanderson, K. \& Cox, G. E. (1998). Pseudomonas aeruginosa as a cause of infectious diarrhoea. Epidemiol Infect 121, 237-241.

Altschul, S. F., Gish, W., Miller, W., Myers, E. W. \& Lipman, D. J. (1990). Basic local alignment search tool. J Mol Biol 215, 403-410.

Balsari, A., Ceccarelli, A., Dubini, F., Fesce, E. \& Poli, G. (1982). The fecal microbial population in the irritable bowel syndrome. Microbiologica 5, 185-194.

Baron, A. D. \& Hollander, H. (1993). Pseudomonas aeruginosa bronchopulmonary infection in late human immunodeficiency virus disease. Am Rev Respir Dis 148, 992-996.

Bleday, R., Braidt, J., Ruoff, K., Shellito, P. C. \& Ackroyd, F. W. (1993). Quantitative cultures of the mucosal-associated bacteria in the mechanically prepared colon and rectum. Dis Colon Rectum 36, 844-849.

Bonten, M. J., Bergmans, D. C., Speijer, H. \& Stobberingh, E. E. (1999). Characteristics of polyclonal endemicity of Pseudomonas aeruginosa colonization in intensive care units. Implications for infection control. Am J Respir Crit Care Med 160, 1212-1219.

Brooks, S. P., McAllister, M., Sandoz, M. \& Kalmokoff, M. L. (2003). Culture-independent phylogenetic analysis of the faecal flora of the rat. Can J Microbiol 49, 589-601.

Collins, S. M., Piche, T. \& Rampal, P. (2001). The putative role of inflammation in the irritable bowel syndrome. Gut 49, 743-745.

Dulon, S., Leduc, D., Cottrell, G. S., D’Alayer, J., Hansen, K. K., Bunnett, N. W., Hollenberg, M. D., Pidard, D. \& Chignard, M. (2005). Pseudomonas aeruginosa elastase disables proteinase-activated receptor 2 in respiratory epithelial cells. Am J Respir Cell Mol Biol 32, 411419.

Eckburg, P. B., Bik, E. M., Bernstein, C. N., Purdom, E., Dethlefsen, L., Sargent, M., Gill, S. R., Nelson, K. E. \& Relman, D. A. (2005). Diversity of the human intestinal microbial flora. Science 308, 16351638.

Edlund, C. \& Nord, C. E. (2000). Effect on the human normal microflora of oral antibiotics for treatment of urinary tract infections. J Antimicrob Chemother 46 (Suppl. 1), 41-48.

Fichtenbaum, C. J., Woeltje, K. F. \& Powderly, W. G. (1994). Serious Pseudomonas aeruginosa infections in patients infected with human immunodeficiency virus: a case-control study. Clin Infect Dis 19, 417422.

Flanagan, J. L., Brodie, E. L., Weng, L., Lynch, S. V., Garcia, O., Brown, R., Hugenholtz, P., DeSantis, T. Z., Andersen, G. L. \& other authors (2007). Loss of bacterial diversity during antibiotic treatment of intubated patients colonized with Pseudomonas aeruginosa. J Clin Microbiol 45, 1954-1962.

Furrie, E. (2006). A molecular revolution in the study of intestinal microflora. Gut 55, 141-143.

George, S. E., Walsh, D. B., Stead, A. G. \& Claxton, L. D. (1989). Effect of ampicillin-induced alterations in murine intestinal microbiota on the survival and competition of environmentally released pseudomonads. Fundam Appl Toxicol 13, 670-680.

Gorbach, S. L., Barza, M., Giuliano, M. \& Jacobus, N. V. (1988). Colonization resistance of the human intestinal microflora: testing the hypothesis in normal volunteers. Eur J Clin Microbiol Infect Dis 7, 98102.

Hentges, D. J., Stein, A. J., Casey, S. W. \& Que, J. U. (1985). Protective role of intestinal flora against infection with Pseudomonas aeruginosa in mice: influence of antibiotics on colonization resistance. Infect Immun 47, 118-122.
Hooper, L. V. \& Gordon, J. I. (2001). Commensal host-bacterial relationships in the gut. Science 292, 1115-1118.

Hopkins, M. J. \& Macfarlane, G. T. (2002). Changes in predominant bacterial populations in human faeces with age and with Clostridium difficile infection. J Med Microbiol 51, 448-454.

Kassinen, A., Krogius-Kurikka, L., Makivuokko, H., Rinttila, T., Paulin, L., Corander, J., Malinen, E., Apajalahti, J. \& Palva, A. (2007). The fecal microbiota of irritable bowel syndrome patients differs significantly from that of healthy subjects. Gastroenterology 133, 24-33.

Kerckhoffs, A. P. M., Samsom, M., van der Rest, M. E., de Vogel, J., Knol, J., Ben-Amor, K. \& Akkermans, L. M. A. (2009). Lower Bifidobacteria counts in both duodenal mucosa-associated and fecal microbiota in irritable bowel syndrome patients assessed by molecular analysis. World J Gastroenterol 15, 2887-2892.

Kessner, D. M. \& Lepper, M. H. (1967). Epidemiologic studies on gram-negative bacilli in the hospital and community. Am J Epidemiol 85, 45-60.

King, T. S., Elia, M. \& Hunter, J. O. (1998). Abnormal colonic fermentation in irritable bowel syndrome. Lancet 352, 1187-1189.

Kühbacher, T., Ott, S. J., Helwig, U., Mimura, T., Rizzello, F., Kleessen, B., Gionchetti, P., Blaut, M., Campieri, M. \& other authors (2006). Bacterial and fungal microbiota in relation to probiotic therapy (VSL\#3) in pouchitis. Gut 55, 833-841.

León-Barúa, R., Gilman, R. H., Rodríguez, C., Bonilla, J. J., Yi, A., Maúrtua, D. \& Sack, R. B. (1993). Comparison of three methods to obtain upper small bowel contents for culture. Am J Gastroenterol 88, 925-928.

Lepage, P., Seksik, P., Sutren, M., de la Cochetiere, M. F., Jian, R., Marteau, P. \& Dore, J. (2005). Biodiversity of the mucosa-associated microbiota is stable along the distal digestive tract in healthy individuals and patients with IBD. Inflamm Bowel Dis 11, 473-480.

Levison, M. E. (1973). Effect of colon flora and short-chain fatty acids on growth in vitro of Pseudomonas aeruginosa and Enterobacteriaceae. Infect Immun 8, 30-35.

Levy, J. (2000). The effects of antibiotic use on gastrointestinal function. Am J Gastroenterol 95, S8-S10.

Li, M., Wang, B., Zhang, M., Rantalainen, M., Wang, S., Zhou, H., Zhang, Y., Shen, J., Pang, X. \& other authors (2008). Symbiotic gut microbes modulate human metabolic phenotypes. Proc Natl Acad Sci U S A 105, 2117-2122.

Liu, W. \& Saint, D. A. (2002a). A new quantitative method of real time reverse transcription polymerase chain reaction assay based on simulation of polymerase chain reaction kinetics. Anal Biochem 302, 52-59.

Liu, W. \& Saint, D. A. (2002b). Validation of a quantitative method for real time PCR kinetics. Biochem Biophys Res Commun 294, 347353.

Livermore, D. M. (1992). Interplay of impermeability and chromosomal beta-lactamase activity in imipenem-resistant Pseudomonas aeruginosa. Antimicrob Agents Chemother 36, 2046-2048.

Madden, J. A. \& Hunter, J. O. (2002). A review of the role of the gut microflora in irritable bowel syndrome and the effects of probiotics. Br J Nutr 88, S67-S72.

Mah, T. F., Pitts, B., Pellock, B., Walker, G. C., Stewart, P. S. \& O'Toole, G. A. (2003). A genetic basis for Pseudomonas aeruginosa biofilm antibiotic resistance. Nature 426, 306-310.

Mai, V. \& Morris, J. G., Jr (2004). Colonic bacterial flora: changing understandings in the molecular age. J Nutr 134, 459-464.

Malinen, E., Rinttila, T., Kajander, K., Matto, J., Kassinen, A., Krogius, L., Saarela, M., Korpela, R. \& Palva, A. (2005). Analysis of the fecal 
microbiota of irritable bowel syndrome patients and healthy controls with real-time PCR. Am J Gastroenterol 100, 373-382.

Masuda, N., Gotoh, N., Ishii, C., Sakagawa, E., Ohya, S. \& Nishino, T. (1999). Interplay between chromosomal $\beta$-lactamase and the MexABOprM efflux system in intrinsic resistance to $\beta$-lactams in Pseudomonas aeruginosa. Antimicrob Agents Chemother 43, 400-402.

Maxwell, P. R., Rink, E., Kumar, D. \& Mendall, M. A. (2002). Antibiotics increase functional abdominal symptoms. Am J Gastroenterol 97, 104108.

Mueller, S., Saunier, K., Hanisch, C., Norin, E., Alm, L., Midtvedt, T., Cresci, A., Silvi, S., Orpianesi, C. \& other authors (2006). Differences in fecal microbiota in different European study populations in relation to age, gender, and country: a cross-sectional study. Appl Environ Microbiol 72, 1027-1033.

Muyzer, G. \& Smalla, K. (1998). Application of denaturing gradient gel electrophoresis (DGGE) and temperature gradient gel electrophoresis (TGGE) in microbial ecology. Antonie van Leeuwenhoek 73, $127-141$

Muyzer, G., de Waal, E. C. \& Uitterlinden, A. G. (1993). Profiling of complex microbial populations by denaturing gradient gel electrophoresis analysis of polymerase chain reaction-amplified genes coding for $16 \mathrm{~S}$ rRNA. Appl Environ Microbiol 59, 695-700.

Nadkarni, M. A., Martin, F. E., Jacques, N. A. \& Hunter, N. (2002). Determination of bacterial load by real-time PCR using a broad-range (universal) probe and primers set. Microbiology 148, 257-266.

Niedzielin, K., Kordecki, H. \& Birkenfeld, B. (2001). A controlled, double-blind, randomized study on the efficacy of Lactobacillus plantarum $299 \mathrm{~V}$ in patients with irritable bowel syndrome. Eur J Gastroenterol Hepatol 13, 1143-1147.

Nobaek, S., Johansson, M. L., Molin, G., Ahrne, S. \& Jeppsson, B. (2000). Alteration of intestinal microflora is associated with reduction in abdominal bloating and pain in patients with irritable bowel syndrome. Am J Gastroenterol 95, 1231-1238.

Oliver, A., Canton, R., Campo, P., Baquero, F. \& Blazquez, J. (2000). High frequency of hypermutable Pseudomonas aeruginosa in cystic fibrosis lung infection. Science 288, 1251-1254.

O'Mahony, L., McCarthy, J., Kelly, P., Hurley, G., Luo, F., Chen, K., O'Sullivan, G. C., Kiely, B., Collins, J. K. \& other authors (2005). Lactobacillus and bifidobacterium in irritable bowel syndrome: symptom responses and relationship to cytokine profiles. Gastroenterology 128, 541-551.

Parry, S. D., Stansfield, R., Jelley, D., Gregory, W., Phillips, E., Barton, J. R. \& Welfare, M. R. (2003). Does bacterial gastroenteritis predispose people to functional gastrointestinal disorders? A prospective, community-based, case-control study. Am J Gastroenterol 98, 19701975.

Pimentel, M., Chow, E. J. \& Lin, H. C. (2000). Eradication of small intestinal bacterial overgrowth reduces symptoms of irritable bowel syndrome. Am J Gastroenterol 95, 3503-3506.
Pirnay, J. P., De Vos, D., Duinslaeger, L., Reper, P., Vandenvelde, C., Cornelis, P. \& Vanderkelen, A. (2000). Quantitation of Pseudomonas aeruginosa in wound biopsy samples: from bacterial culture to rapid 'real-time' polymerase chain reaction. Crit Care 4, 255-261.

Posserud, I., Stotzer, P. O., Bjornsson, E. S., Abrahamsson, H. \& Simrén, M. (2007). Small intestinal bacterial overgrowth in patients with irritable bowel syndrome. Gut 56, 802-808.

Quigley, E. M. (2007). Probiotics in irritable bowel syndrome: an immunomodulatory strategy? J Am Coll Nutr 26, 684S-690S.

Rodríguez, L. A. \& Ruigómez, A. (1999). Increased risk of irritable bowel syndrome after bacterial gastroenteritis: cohort study. BMJ 318, 565-566.

Sanguinetti, C. J., Dias, N. E. \& Simpson, A. J. (1994). Rapid silver staining and recovery of PCR products separated on polyacrylamide gels. Biotechniques 17, 914-921.

Si, J. M., Yu, Y. C., Fan, Y. J. \& Chen, S. J. (2004). Intestinal microecology and quality of life in irritable bowel syndrome patients. World J Gastroenterol 10, 1802-1805.

Speert, D. P., Campbell, M. E., Davidson, A. G. \& Wong, L. T. (1993). Pseudomonas aeruginosa colonization of the gastrointestinal tract in patients with cystic fibrosis. J Infect Dis 167, 226-229.

Spiller, R. C. (2007). Role of infection in irritable bowel syndrome. J Gastroenterol 42 (Suppl. 17), 41-47.

Suau, A., Bonnet, R., Sutren, M., Godon, J. J., Gibson, G. R., Collins, M. D. \& Dore, J. (1999). Direct analysis of genes encoding $16 \mathrm{~S}$ rRNA from complex communities reveals many novel molecular species within the human gut. Appl Environ Microbiol 65, 4799-4807.

Vaughan, E. E., Schut, F., Heilig, H. G., Zoetendal, E. G., de Vos, W. M. \& Akkermans, A. D. (2000). A molecular view of the intestinal ecosystem. Curr Issues Intest Microbiol 1, 1-12.

Vergnolle, N. (2005). Clinical relevance of proteinase activated receptors (pars) in the gut. Gut 54, 867-874.

Walters, M. C., III, Roe, F., Bugnicourt, A., Franklin, M. J. \& Stewart, P. S. (2003). Contributions of antibiotic penetration, oxygen limitation, and low metabolic activity to tolerance of Pseudomonas aeruginosa biofilms to ciprofloxacin and tobramycin. Antimicrob Agents Chemother 47, 317-323.

Woodmansey, E. J., McMurdo, M. E., Macfarlane, G. T. \& Macfarlane, S. (2004). Comparison of compositions and metabolic activities of fecal microbiotas in young adults and in antibiotic-treated and nonantibiotic-treated elderly subjects. Appl Environ Microbiol 70, 61136122.

Zhang, Z., Schwartz, S., Wagner, L. \& Miller, W. (2000). A greedy algorithm for aligning DNA sequences. J Comput Biol 7, 203-214.

Zoetendal, E. G., von Wright, A., Vilpponen-Salmela, T., Ben Amor, K., Akkermans, A. D. \& de Vos, W. M. (2002). Mucosa-associated bacteria in the human gastrointestinal tract are uniformly distributed along the colon and differ from the community recovered from feces. Appl Environ Microbiol 68, 3401-3407. 